\title{
Grinding MARAging STEel VACO 180 WITH SiC GRINDING WHEELS - GRINDING FORCE AND WHEEL WEAR
}

\author{
Farský Jindřich, Bakša Tomáš, Zetek Miroslav
}
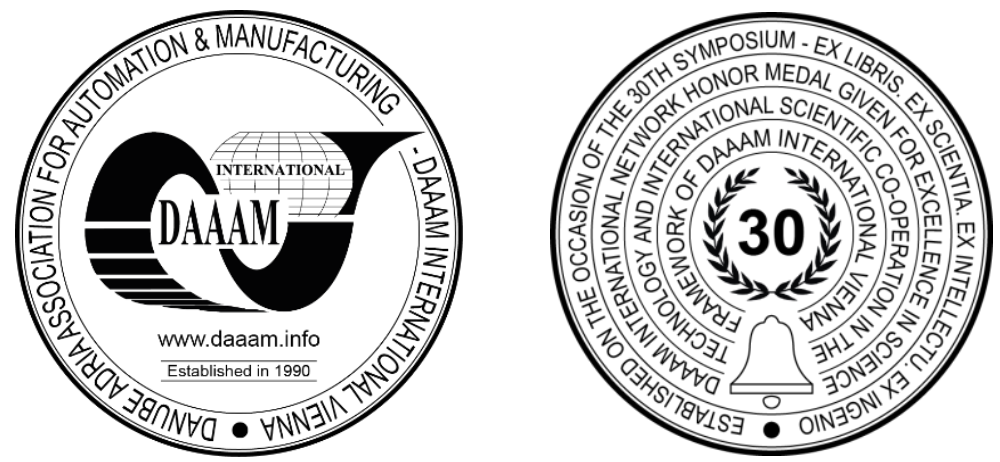

This Publication has to be referred as: Farsky, J[indrich]; Baksa, T[omas] \& Zetek, M[iroslav] (2019). Grinding of Maraging Steel VACO 180 with SiC Grinding Wheels - Grinding Force and Wheel Wear, Proceedings of the 30th DAAAM International Symposium, pp.0641-0645, B. Katalinic (Ed.), Published by DAAAM International, ISBN 9783-902734-22-8, ISSN 1726-9679, Vienna, Austria

DOI: $10.2507 / 30$ th.daaam.proceedings.088

\begin{abstract}
Grinding is a technology which is used as a surface finishing operation. Therefore it is important for achieving good dimensions of accuracy. This accuracy can be affected by the size of wear of the grinding wheel. The size of the grinding wheel wear can be affected by the size of the grinding forces. Both of these factors can be influenced by the grinding parameters and the grinding wheel composition. Therefore, this article focuses on the influence of grinding parameters on the wear of grinding wheels and the size of the grinding forces when grinding VACO 180 maraging steel using SiC grinding wheels.
\end{abstract}

Keywords: Grinding; Grinding wheels; SiC, Forces; Wearing; Maraging steel

\section{Introduction}

Grinding is one of the finishing operations used in the industry where it is necessary to achieve a good surface quality, high dimensional accuracy and also for materials which cannot be machined using other types of cutting technologies with the defined geometry of tools. [1] In addition to this, grinding is used to finish the surfaces of test specimens, when these areas of specimens have high requirements for precision and minimal impact from grinding forces. In order to achieve these requirements, it is necessary to select a suitable grinding wheel with suitable cutting conditions for the test material. The grinding speed and depth of cut are the most influential parameters on the results of grinding, as is shown in Fig. 1. From this figure it is evident that changing these two parameters can influence the size of wear, grinding forces, the temperature of grinding, cutting time and so on. [2], [3], [4], [5], [6]

This paper deals with grinding VACO 180, a material which is used for producing test specimens for mechanical tests. This is because it is equivalent to the material according to DIN X3NiCoMo 189 5, but mainly to MS1 steel from EOS, which is used for metal 3D printing. As a result, it is then possible to compare the differences between the properties of $3 \mathrm{D}$ printed and conventional material. The chemical composition and mechanical properties of VACO 180 are given in Table 1 and Table 2. [7] 


\begin{tabular}{|c|c|c|c|c|c|}
\hline C & Co & Mo & Ni & Ti & Others \\
\hline$<0.03 \%$ & $9.0 \%$ & $5.0 \%$ & $18.5 \%$ & $0.75 \%$ & $\mathrm{Al}, \mathrm{B}, \mathrm{Zr}$ \\
\hline
\end{tabular}

Table 1. Chemical composition of VACO 180 [7]

\begin{tabular}{|c|c|c|c|c|c|}
\hline Yield strength & Tensile strength & Ductility & Contraction & Notch toughness & Hardness \\
\hline $640 \mathrm{MPa}$ & $930-1130 \mathrm{MPa}$ & $12 \%$ & $60 \%$ & $55 \mathrm{~J}$ & $350 \mathrm{HV}$ \\
\hline
\end{tabular}

Table 2. Mechanical properties of VACO 180 [7]

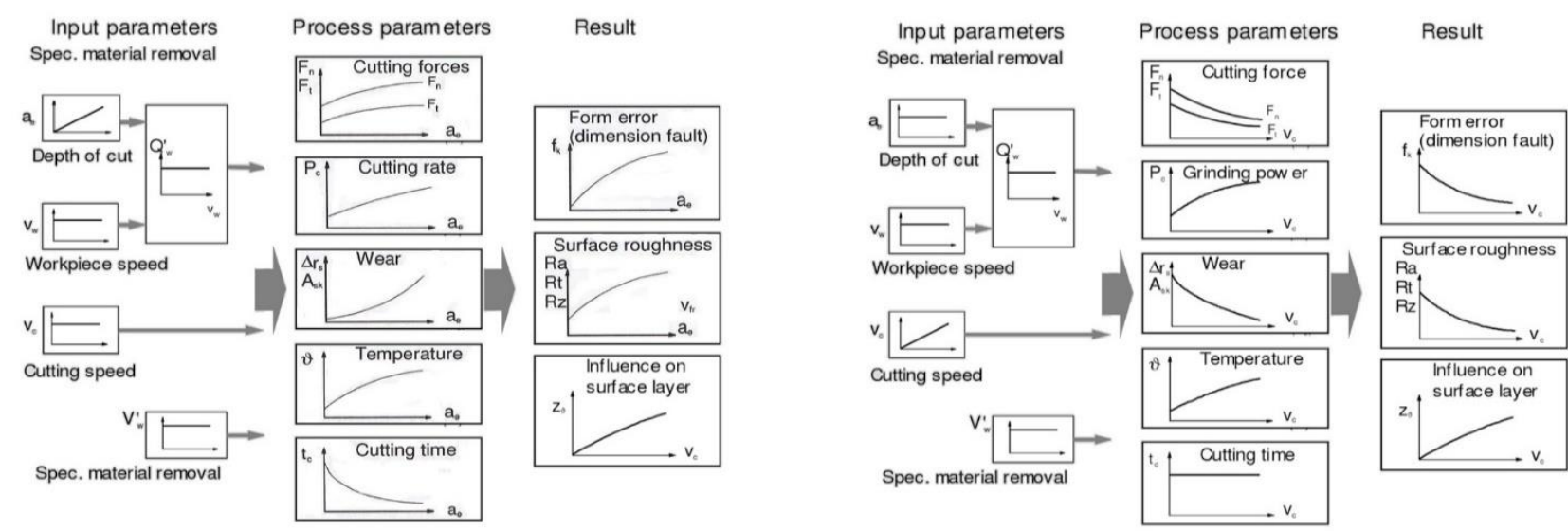

Fig. 1. Influence of grinding parameters on the results [2]

\section{Experiment}

These experiments are derived from past research which was focused on grinding this material with $\mathrm{SiC}$ grinding wheels. From the past experiments, the results were not satisfactory in terms of the grinding wheels used. Therefore, for these experiments a different composition of grinding wheels was used. The grinding wheels used and their compositions are shown in Table 3. Differences between them are in the grain size and hardness. Flat bars with dimensions $36 \mathrm{x} 5.2 \mathrm{x}$ $180 \mathrm{~mm}$ (height $\mathrm{x}$ width $\mathrm{x}$ length) were used as test specimens. After grinding the face was $5.2 \mathrm{x} 180 \mathrm{~mm}$. The width of the grinding wheel was bigger than the width of the test specimen. Experimental grinding was carried out on the ANCA mx7 tool grinding machine which had to be fitted with a special clamp system for the flat bars. [8], [9]

\begin{tabular}{|c|c|c|c|c|c|c|c|}
\hline Designation & Specification & Shape & Dimension & Type of grain & $\begin{array}{c}\text { Grain size } \\
\text { [FEPA] }\end{array}$ & Bonding & Hardness \\
\hline $\mathrm{SiC}-240$ & $\begin{array}{c}49 \mathrm{C} 240 \mathrm{~J} 10 \\
\mathrm{~V} 40\end{array}$ & $1 \mathrm{~A} 1$ & $100 \times 10$ & $\begin{array}{c}\text { Silicon } \\
\text { carbide green }\end{array}$ & 240 (very soft) & Ceramic & soft \\
\hline SiC-80 & $\begin{array}{c}49 \mathrm{C} 80 \mathrm{~K} 9 \\
\mathrm{~V} 40\end{array}$ & $1 \mathrm{~A} 1$ & $100 \times 10$ & $\begin{array}{c}\text { Silicon } \\
\text { carbide green }\end{array}$ & 80 (soft) & Ceramic & medium \\
\hline
\end{tabular}

Table 3. Used grinding wheels

The next set of variables for these experiments are the grinding conditions. As there are many grinding conditions it was necessary to choose only those which have the biggest influence on the force and wheel wear. Grinding speed and depth of cut were chosen as the variables for these experiments. The values of these variables are shown in Table xx. The next value of the grinding conditions is wheel speed which was $200 \mathrm{~mm} . \mathrm{min}^{-1}$. This value was the same for each experiment. The experiments were carried out using coolant which was directed on to the surface of the grinding wheel using two nozzles. Both nozzles were pointed at the contact point of the grinding wheel with the surface of the test specimens. The technology for grinding in these experiments is shown in Table 4.

\begin{tabular}{|c|c|c|c|c|c|c|c|c|}
\hline Designation & $v_{c}\left[m_{0} s^{-1}\right]$ & $\mathbf{a e}[\mathrm{mm}]$ & Designation & $\mathrm{Vc}\left[\mathrm{m} \cdot \mathrm{s}^{-1}\right]$ & $\mathrm{ae}[\mathrm{mm}]$ & Designation & $\mathrm{vc}_{\mathrm{c}}\left[\mathrm{m} . \mathrm{s}^{-1}\right]$ & $\mathrm{ae}_{\mathrm{e}}[\mathrm{mm}]$ \\
\hline Exp01 & 25 & 0.025 & Exp04 & 25 & 0.05 & Exp07 & 25 & 0.1 \\
\hline Exp02 & 30 & 0.025 & Exp05 & 30 & 0.05 & Exp08 & 30 & 0.1 \\
\hline Exp03 & 35 & 0.025 & Exp06 & 35 & 0.05 & Exp09 & 35 & 0.1 \\
\hline
\end{tabular}

Table 4. Used grinding conditions for experiments

\section{Results}

The experiments focused on the size of forces and wear of the grinding wheels during grinding. As the ANCA mx7 grinder can record only the percentage value of the spindle load it was necessary to recalculate these loads to forces. Also, it was necessary to subtract the values of centrifugal loads. The formula for recalculating percentage spindle loads to forces is shown in (1). 


$$
F=\frac{P \cdot p_{p}}{2 \cdot 100 \cdot \pi \cdot n \cdot r}[N]
$$

$\begin{array}{llll}\mathrm{F} & \ldots & \text { Force } & {[\mathrm{N}]} \\ \mathrm{p} p & \ldots & \text { Percentage spindle load } & {[\%]} \\ \mathrm{P} & \ldots & \text { Power of spindle } & {[\mathrm{W}]} \\ \mathrm{r} & \ldots & \text { Radius of grinding wheel } & {[\mathrm{m}]} \\ \mathrm{n} & \ldots & \text { revolutions per minute } & {[\mathrm{rpm}]}\end{array}$

These recalculated values are shown in the first two graphs (Fig. 2 and Fig. 3). The first graph shows the force values for the SiC-240 grinding wheel and the second one is for SiC-80. It is immediately clear that grinding wheel SiC-240, which is softer, created smaller forces than the second one. If we look at the first graph (Fig. 2) we can see the forces decrease only for the depth of cut $0.025 \mathrm{~mm}$ depending on the increasing grinding speed. At other cut depths, the forces decrease too, but then they stabilise or increase. If we look at the results from the second grinding wheel (Fig. 3) we can see the size of forces is higher than the first one. This is caused by the hardness of the grinding wheel which is higher because the tougher structure of wheel puts more resistance on the grinding process. In this grinding wheel, we can see the forces decrease with the higher grinding speed at all depths of cuts.

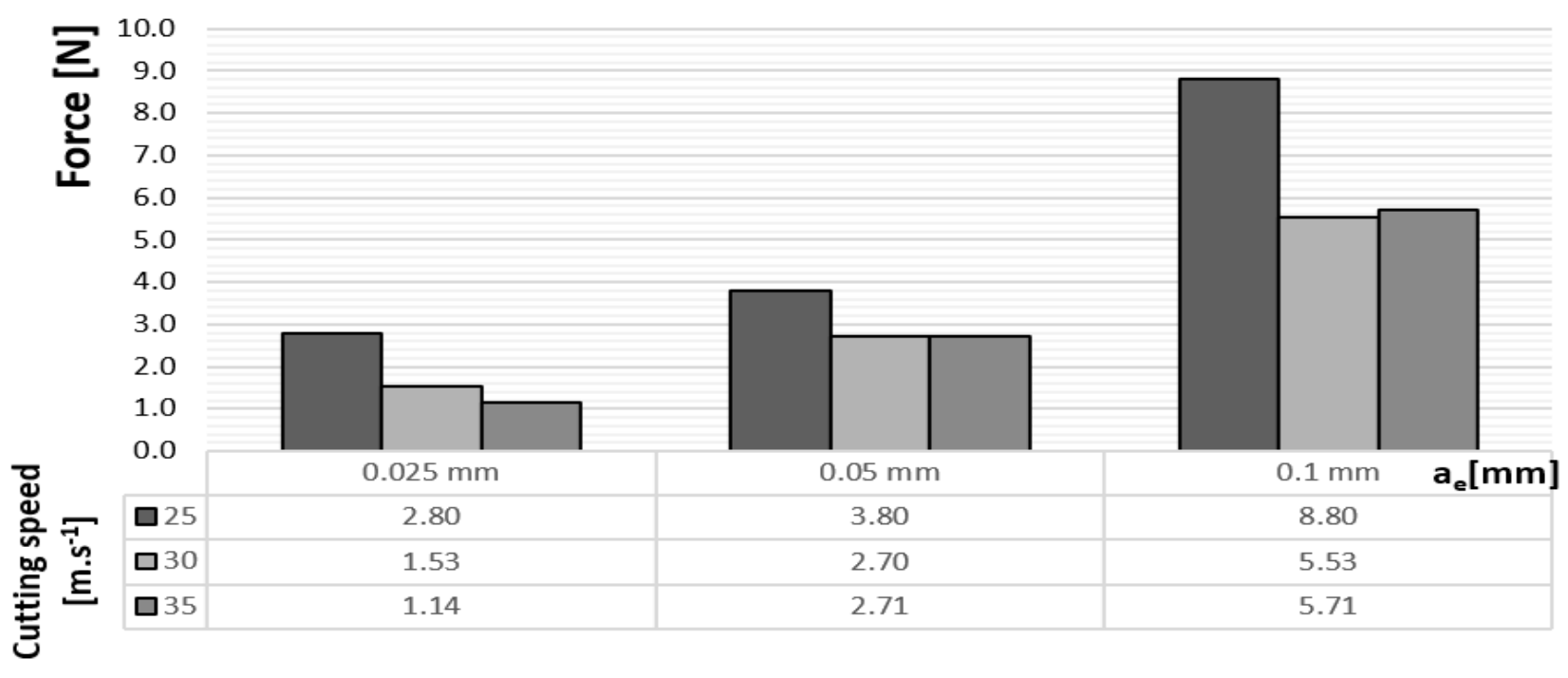

Fig. 2. The size of forces from grinding wheel $\mathrm{SiC}-240$

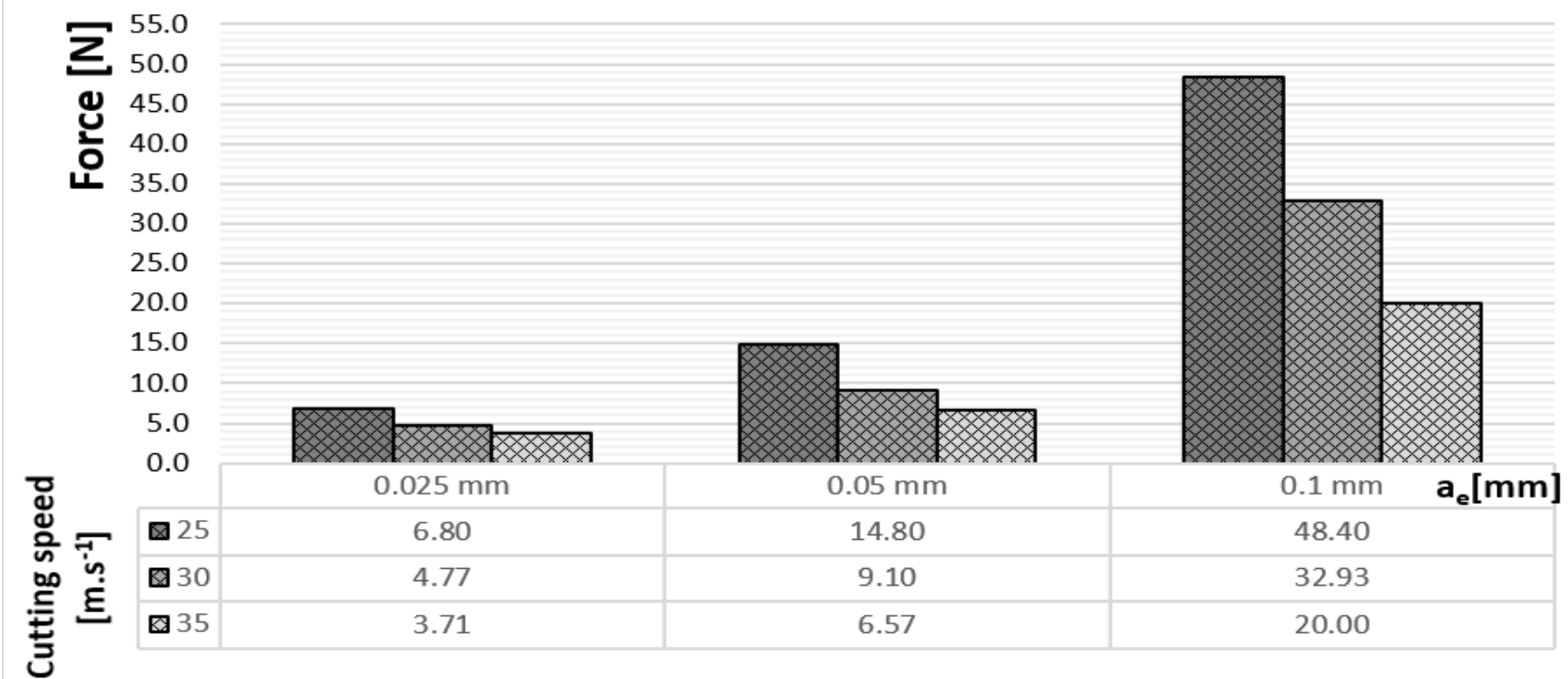

Fig. 3. The size of forces from grinding wheel SiC-80 
Fig.4 shows the results from the wear of both grinding wheels. The SiC - 240 grinding wheel is without cross fill and the SiC - 80 grinding wheel is with cross fill. We can see a big difference between the grinding wheels. Also, these big differences are visible between different grinding conditions. The SiC-240 grinding wheel showed higher values of wear than the second one. Also, when the grinding speed was increased there was an increase in wear of the grinding wheel. On the second grinding wheel, SiC-80, there was a smaller size of wear than on the first one. Also, the course of wear, in this case, decreased with increasing grinding speed. In both cases, the wear increased when the depth of cut was higher.

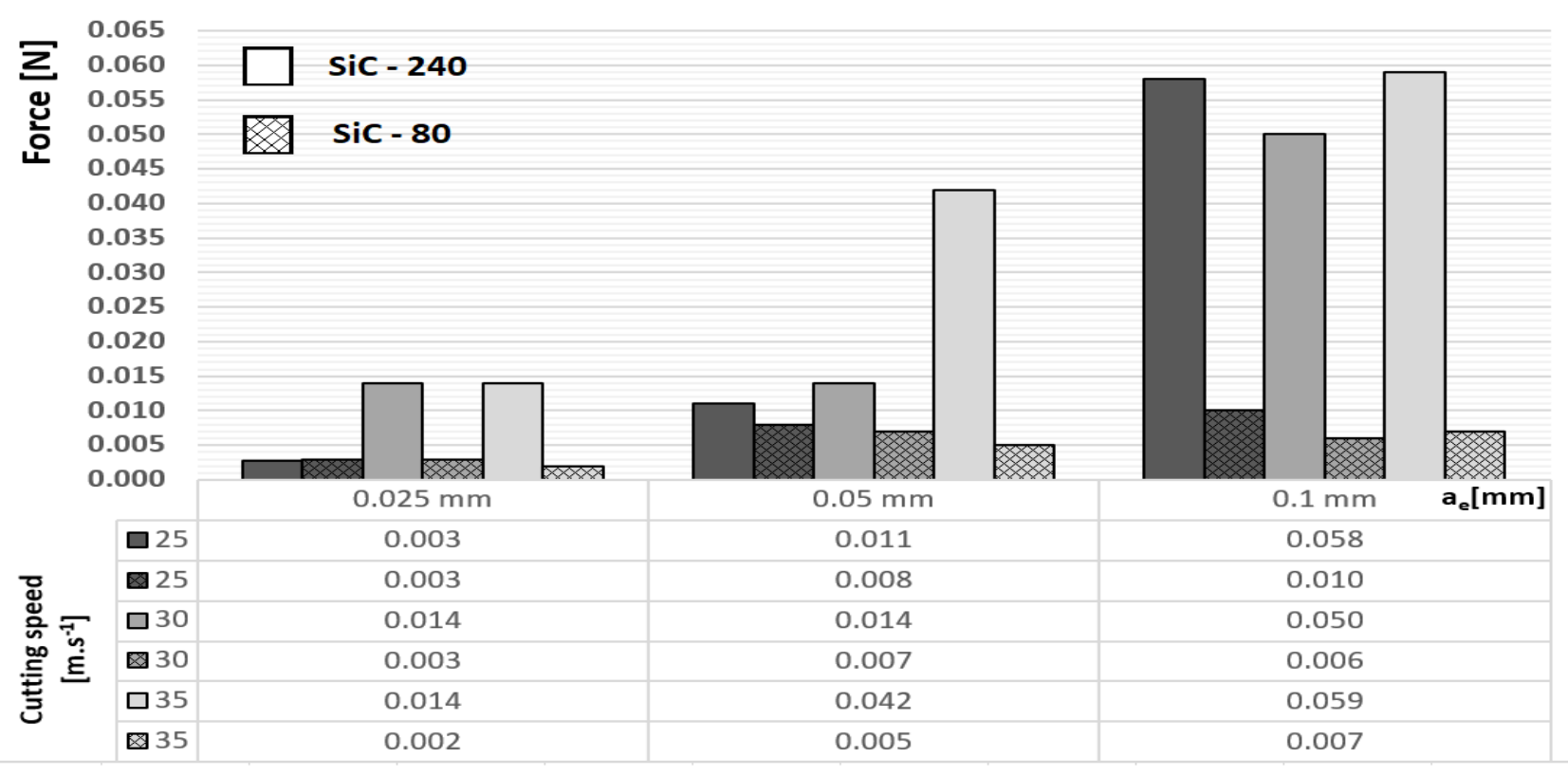

Fig. 4. The size of wear on SiC-240 and SiC-80 grinding wheels

When we compare the results of wear and grinding forces we can see dependence especially for the second grinding wheel SiC-80. In this grinding wheel can be seen the wear of the grinding wheel decreasing when decreasing the size of the grinding forces. The first grinding wheel SiC-240 does not have this dependency. This may be caused by an unexpected change in the grinding forces depending on the grinding speed.

\section{Conclusion}

This work focused on investigating the influence of grinding speed and depth of cut on the size of grinding forces and wear for two grinding wheels with different structures. From the results it is evident that the SiC-240 grinding wheel has the smallest size of grinding forces but the changes to these forces were undesirable in relation to the increase of the grinding speed. Also, it is related to the changes of wear of this grinding wheel, which was similar. The results of the second grinding wheel SiC-80 reach higher values of forces but smaller values of wear and also a better course for both cases. From these results it is obvious that the second grinding wheel is better for these applications even if bigger values of forces are reached, but these values are still small enough. This grinding wheel also reached smaller values of wear.

These experiments serve for future research of the usability of grinding wheels for grinding VACO 180 maraging steel. In the next step, we plan to use different sized grinding wheels with this structure and focus also on results of surface quality which is very important in our research.

\section{Acknowledgments}

This paper was supported by the Internal Grant Agency of the University of West Bohemia, project No. SGS-2019-008 Research and Development for Innovation in the Field of Manufacturing Technology - Machining Technology III.

\section{References}

[1] K. Kannan and N. Arunachalam, 'Grinding wheel redress life estimation using force and surface texture analysis', Procedia CIRP, vol. 72, pp. 1439-1444, ISNN: 2212-8271 Jan. 2018.

[2] F. Klocke, Manufacturing Processes 2: Grinding, Honing, Lapping. Springer Science \& Business Media, 2009.

[3] J. P. Davim, Modern Machining Technology: A Practical Guide. Elsevier, 2011.

[4] R. Dubovska, J. Jambor, and J. Majerik, 'Qualitative Aspects of Machined Surfaces of High Strength Steels', Procedia Eng., vol. 69, no. Supplement C, pp. 646-654, ISNN: 1877-7058 Jan. 2014. 
[5] T. Baksa, O. Hronek, J. Farsky, and M. Zetek, 'Influence of cutting conditions on the surface quality and grinding wheel wear during cylindrical grinding of inconel 718', presented at the Annals of DAAAM and Proceedings of the International DAAAM Symposium, 2017, pp. 500-505, ISBN: 978-390273411-2.

[6] L. Rokyta, 'Testing of Grinding Wheels For Tools Manufacturing', in Annals of DAAAM and Proceedings of the International DAAAM Symposium, vol. 2011, ISBN: 978-3-901509-83-4.

[7] 'DataSheet maraging steel - VACO 180', Bolzano. [Online]. Available: https://www.bolzano.cz/assets/files/materialove_listy/Vaco\%20180(1).pdf. [Accessed: 25-Sep-2019].

[8] T. Bakša, J. Farský, O. Hronek, and M. Zetek, 'Influence of depth of cut on quality of ground surface and cutting force when grinding cermet', Manuf. Technol., vol. 18, no. 3, pp. 352-356, ISNN: 12132489, 2018.

[9] H. K. Tönshoff, T. Friemuth, and J. C. Becker, 'Process monitoring in grinding', CIRP Ann. - Manuf. Technol., vol. 51, no. 2, pp. 551-571, ISNN: 00078506, 2002. 